\title{
The topaz micro-radiofrequency ablation achieves similar results to endoscopic plantar fascia release
}

\begin{abstract}
Plantar fasciitis is usually responsive to conservative treatment. Recalcitrant cases might require surgery. The current study is a retrospective evaluation of two surgical techniques used to treat plantar fasciitis. Endoscopic plantar fascia release and micro-radiofrequency ablation (TOPAZ) were used in our department over the last decade. The patients were all treated conservatively according to a standardized protocol. Failed cases were operated using either technique. The current study evaluates the results of the treatment. There were 24 TOPAZ procedures and 39 endoscopic plantar fascia releases, choice of treatment was not randomized and depended on surgeon's assessment. Pain levels declined from $6.8 \pm 1.5$ to $1.8 \pm 1.5$ on the VAS scale.

The results in both groups were similar. It is possible to conclude that surgical treatment of plantar fasciitis is an efficient procedure with relatively gratifying results. Complications tend to be slightly lower in the TOPAZ group.
\end{abstract}

\author{
Volume 9 Issue 4 - 2017
}

\section{Mustafa Yassin, Avraham Garti, Eyal Heller, Dror Robinson \\ Department of Orthopaedic Surgery, Hasharon Hospital, Rabin} Medical Center, Tel Aviv University Medical School, Israel

Correspondence: Dror Robinson MD, Department of Orthopedics, Hasharon Hospital, Petah Tikwa, Israel, Tel 972543342899, Fax 972-892060।3, Email dror6 I@gmail.com

Received: November 27, 2017 | Published: December 06, 2017

\section{Introduction}

Plantar fasciitis lifetime prevalence is approximately $10 \%$ of the US population creating a significant burden to the health care delivery system. Approximately $90 \%$ of patients diagnosed with plantar fasciitis respond to nonsurgical therapy and do not require surgical intervention. ${ }^{1}$ However plantar fasciitis has a significant deleterious effect on both foot function and general health overall functional score. ${ }^{2}$ Suspected etiologies ${ }^{3-5}$ include obesity, overuse due to walking or running or standing, excessive subtalar pronation, seronegative arthritis, and limited dorsiflexion of the ankle joint. Conservative therapy usually entails the use of foot orthoses, stretching exercises, local corticosteroid injection, oral nonsteroidal anti-inflammatory drugs and other physical therapy modalities, and nonweight-bearing status and rest.

The small subset that requires surgery are patients in whom 6 months or more of conservative therapy has not led to remission. Open fasciotomy, has a high satisfaction rate but a prolonged recovery period. ${ }^{6}$ Endoscopic plantar fasciotomy allows faster return to regular activities in comparison with open surgical approaches. ${ }^{6}$ Risks associated with plantar fascia surgery include complex regional pain syndrome, ${ }^{7}$ nerve damage and lateral column collapse., ${ }^{7,8}$

These complications of surgical approaches create an interest in minimal invasive interventions, these include extracorporeal shockwave therapy that is similar in its efficacy to placebo, ${ }^{9}$ cryosurgery appears to be somewhat more successful with a $76 \%$ success rate. ${ }^{10}$

The current study was performed in order to try to determine the effectiveness and safety of minimally invasive percutaneous bipolar radiofrequency plantar fasciotomy for the treatment of recalcitrant plantar fasciitis (fasciosis).

The study compared a consecutive series of patients treated by either endoscopic assisted fasciotomy or with radiofrequence ablation of the plantar fascia. The study hypothesis was that percutaneous radiofrequency plantar fasciotomy would demonstrate effectiveness without excessive postoperative complications and with rapid recovery in comparison with an endoscopic surgical approach to this common pedal pathology.

\section{Patients and methods}

\section{Patient population}

The patients were a consecutive series of patients selected from the authors' surgical records from January 2007 to May 2016 at a public hospital.

\section{Plantar fasciitis diagnosis}

The diagnosis of plantar fasciitis was made based on all below described four factors presence:

A. Patient's history with typical pain during the first few steps

B. Physical examination, including the following positive findings:

a. Focal proximal plantar fascia tenderness upon deep palpation.

b. Plantarmedial heel pain without distinct neuritic radiation.

C. Sonographic evaluation of the plantar fascia (minimal $5 \mathrm{~mm}$ thickness).

D. Standard foot standing radiographs (demonstrating a dorsal spur of at least $2 \mathrm{~mm}$ size in a lateral standing foot radiographs).

\section{Inclusion criteria}

A. Diagnosis of plantar fasciitis as described above

B. At least 5 on the VAS pain scale $(10=$ maximal possible pain $)$

C. Failure of a minimum of 6 months of conservative therapy before they had surgery. Conservative therapy included at least the following modalities:

a. Including plantar fascia rest taping,

b. Calf and arch stretching,

c. Functional foot orthotics,

d. At least two local corticosteroid injections,

e. At least a two weeks course of oral etoricoxib $90 \mathrm{mg}$ per day

f. At least two weeks course of topical diclofenac cream $1 \%$. 
Plantar fasciitis is sometimes confused with other diagnoses. In order to rule out other diagnosis the following exclusion criteria were defined:

\section{A. Known sciatica}

B. Positive straight leg raise,

C. Signs of peripheral neuropathy such as loss of sensation in the toes or the foot,

D. Previously diagnosed rheumatoid or other inflammatory arthririts,

E. Calcaneal bone tumor,

F. Positive tarsal tunnel Tinnel's test.

The surgeons evaluated the participants both preoperatively and the postoperatively. A research assistant procured the data for study analyses.

Each patient provided answers to the American Orthopaedic Foot \& Ankle Society (AOFAS) hindfoot scoring scale (15) and a Visual Analogue Score, and the answers to 2 additional questions were also required of each patient, specifically: "Would you recommend this surgery to a family member or friend?" and "Would you do the surgery over again, if the heel pain returned?"

\section{Radiofrequency ablation surgical procedure}

Radiofrequency plantar fasciotomy was performed as an isolated surgical procedure in every case. Before administration of anesthesia, the area of maximum tenderness was marked on the foot. After the area was marked, an ankle block was performed using local anesthetic (20 $\mathrm{ml}$ bupivacaine $0.25 \%$ and $20 \mathrm{ml}$ esracaine $1 \%$ ). The foot was then prepped and draped in the usual sterile manner, after which a skin marker was used to draw out a grid of 16 to 20 dots over the proximal plantar fascia, encompassing the point of maximum tenderness, each mark being 3 to $4 \mathrm{~mm}$ apart. Thereafter, an 18-gauge needle was used to puncture through the skin and superficial fascia and subcutaneous fat layers down to the level of the deep fascia. A mosquito clamp was used to enlarge the channel and prevent skin injury. After creation of the channels, the bipolar radiofrequency unit (Stryker SERFAS Energy RF Ablation System, San Jose, CA 95138) was set to a power level of 4 for 20 seconds. Thereafter, the probe was inserted sequentially into each needle channel, to the level of the plantar fascia, which is identified by palpable resistance to penetration by the probe. Once the deep fascia was contacted, the radiofrequency energy was applied, and the probe felt to pierce the deep plantar fascia, thereby effecting microfasciotomy (Figure 1) while the great toe was held at maximal dorsiflexion to tighten the fascia.

Care was taken to avoid penetration through the intrinsic musculature deep to the plantar fascia, and also to avoid application of the radiofrequency energy to the skin. After completion of the microfasciotomy, a sterile CobanTM dressing was applied and the patient placed in a postop shoe and allowed to bear weight to tolerance for 4 weeks postoperatively, regular shoe gear and weight-bearing activities were allowed by 4 weeks postop.

\section{Endoscopic plantar fasciotomy}

Endoscopic plantar fasciotomy was performed as an isolated surgical procedure in every case. Before administration of anesthesia, the area of maximum tenderness was marked on the foot. After the area was marked, an ankle block was performed using local anesthetic (20 $\mathrm{ml}$ bupivacaine $0.25 \%$ and $20 \mathrm{ml}$ esracaine $1 \%$ ). The foot was then prepped and draped in the usual sterile manner. Thereafter, an 18 -gauge needle was used to define the suprafascial plane on the medial side of the heel. A medial portal for $2.7 \mathrm{~mm}$ arthroscopy was created and the camera inserted. A potential space is inflated with 40 $\mathrm{ml}$ of saline in order to allow visualization. Camera rotation allows appreciation of an operative muscle filled potential triangular space. The apex is composed of the bony spur, one side is the plantar fascia (internal surface) and the other is the calcaneal bone (inferior surface). Insertion of a hook allows definition of the width of the plantar fascia and measurement of the width of the medial two thirds of the fascia. The hook is retrieved and a meniscotome is inserted to the measured depth. The arthroscope is used to evaluate the completeness of the fascia release. The great toe was held at maximal dorsiflexion to tighten the fascia.
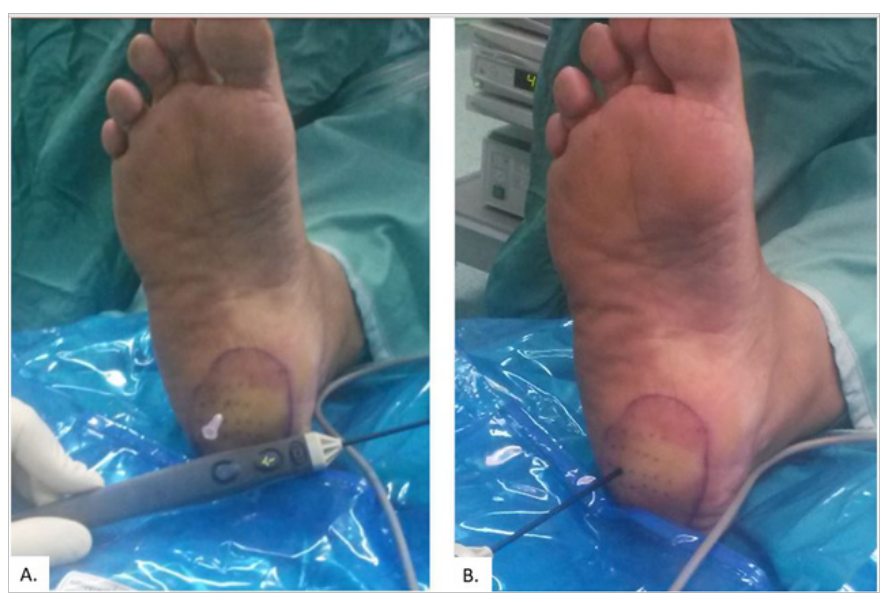

Figure I The operative sequence of the TOPAZ radiofrequency microfasciotomy consists of marking the painful area and filling the area with dots with about $4 \mathrm{~mm}$ separation, an 18 gauge needle is used to penetrate the skin at every dot (A.) then the radiofrequency probe is inserted (B.) (Serfas system, by Stryker).

Care was taken to avoid penetration through the intrinsic musculature deep to the plantar fascia. After completion of the microfasciotomy, a single suture is used to close the portal, a sterile dressing was applied and the patient placed in a postop shoe and allowed to bear weight to tolerance for 4 weeks postoperativelyc regular shoe gear and weight-bearing activities were allowed by 4 weeks postop.

\section{Statistical analysis}

Quantitative variables were compared using the Analyse-it3 software program. The Student's t-test was used, and a significant difference was defined at the 0.05 level. Qualitative variables were analyzed using Wilcoxon-Mann-Whitney non-parametric test.

\section{Results}

A total of 63 eligible patients were identified and their records assessed in this retrospective study. All patients were followed up at the foot $\&$ ankle clinic. A statistical description of the results is depicted in Table 1. The mean age of the patients was 55 years (range, 25-84 years). In regard to gender, 47 of the patients were female $(75 \%)$ and $16(25 \%)$ were male. No bilateral procedures were performed however six patients $(20 \%)$ underwent the intervention on both feet each at a separate surgical setting (with at least a 3-months inter-procedure temporal displacement). 
Table I Demographic data of patients undergoing plantar fascia release and results

\begin{tabular}{|c|c|c|c|}
\hline Parameter & $\begin{array}{l}\text { Endoscopic } \\
\text { Group }\end{array}$ & $\begin{array}{l}\text { Micro-radiofrequency Ablation } \\
\text { Group }\end{array}$ & $\begin{array}{l}\text { Statistical } \\
\text { Significance }\end{array}$ \\
\hline Patient Number & 39 & 24 & \\
\hline Age (Years \pm S.D) & $51.5 \pm 7$ & $61 \pm 8$ & n.s. \\
\hline BMI & $33 \pm 7$ & $36 \pm 9$ & n.s. \\
\hline Gender & $18 \%$ male & $21 \%$ male & n.s. \\
\hline Pre-Op AFAOS Score & $63 \pm 11$ & $48 \pm 10$ & $\mathrm{P}<0.002$ \\
\hline Pre-Op VAS pain Severity Score (I0-maximal pain) & $6.1 \pm 2.1$ & $7.2 \pm 1.8$ & $\mathrm{p}<0.005$ \\
\hline 24 Months AFAOS Score & $85 \pm 5$ & $83 \pm 6$ & n.s. \\
\hline 24 Months VAS pain Severity Score & $1.9 \pm 1.3$ & $1.5 \pm 0.8$ & n.s. \\
\hline AFAOS Score Delta Change & $3 I \pm 9.8$ & $43 \pm 8.6$ & $P<0.001$ \\
\hline$\%$ of patients who recommend the procedure to a friend & $83 \%$ & $78 \%$ & n.s. \\
\hline $\begin{array}{l}\% \text { of patients who would choose to undergo the procedure again if } \\
\text { symptoms recur }\end{array}$ & $62 \%$ & $66 \%$ & n.s. \\
\hline
\end{tabular}

BMI: Body Mass Index

VAS:Visual Analogue Scale, 0-no pain, 10 maximal pain.

The mean duration of pre-operative symptoms was 9 months (range, 6-36 months). The mean duration of postoperative followup was 30 months (range 24-50 months), and almost all of the cases returned to regular shoe gear by 4 weeks after the operation (the one exception was an endoscopic release case with nerve injury).

In regard to the outcome measures, the overall mean of both groups' preoperative AOFAS hindfoot-ankle score was $57 \pm 13$ (range, 42-84). The pre-operative VAS score $(0=$ no pain, $10=$ maximal possible pain $)$ was $6.8 \pm 1.5$ (range, 9-5). At the latest follow-up visit (minimum 24 months) the average AOFAS score was $84.6 \pm 6$ (range, 100-73) and the VAS was $2.8 \pm 1.5$ (range, 5-0).

Regarding recommendation to friends and family, 51 (81\%) of the patients indicated that they would recommend the procedure to a family member or friend, and 40/63 (64\%) of the patients indicated that they would undergo the procedure again should their symptoms recur (the intergroup responses were similar, Fisher exact test).

In regard to complications, the most prevalent complaint expressed by the patients in the postoperative period was that of loss of sensation or electric currents like feeling distal to the operative site in the endoscopy group $(3 / 39,7.6 \%)$. None of the TOPAZ patients, however, developed such symptoms ( $\mathrm{p}<0.05$, Fisher exact test).

Lateral side foot pain occurred in 2 patients in the endoscopy group and in none of the patients in the TOPAZ group (n.s. Fisher exact test).

\section{Discussion}

Plantar fasciitis as a cause of heel pain is associated with thickening of the plantar fascia. ${ }^{11}$ It has been suggested that this thickening can best be observed on a non-weight bearing lateral foot radiograph, ${ }^{12}$ though ultrasonography is often used (as in this study) due to the ability to quantitatively define the plantar fascia thickness. ${ }^{13}$

The spur is measured on lateral radiographs, and a minimal size of 2 millimeters was required to include in this series. While plantar fasciitis is self-limited it is rather disabling, in fact it can be surmised that this disease entity has had significant influence on the quality of life of afflicted individuals. ${ }^{2}$ A surgical indication exists after failure of at least 6 months of conservative therapy.

Conventional surgery is often associated with relatively high morbidity. ${ }^{6}$ This led to the development of multiple procedures for symptomatic relief, including cryosurgery, ${ }^{10}$ botulinum toxin injection, ${ }^{14}$ and extra-corporeal shock wave therapy. ${ }^{9}$ Currently a commonly used technique is endoscopic release of the fascia, ${ }^{8}$ but recently a percutaneous novel radiofrequency based technique has been described. ${ }^{15}$ The main advantage of the percutaneous technique might be reduction in the occurrence of neuropraxia after open and endoscopic interventions, ${ }^{16}$ CRPS,${ }^{7}$ lateral column syndrome, as well as iatrogenic pes planus. ${ }^{17}$ The current study confirms that none of these complications was observed after microfasciotomy via bipolar radiofrequency.

Weil et al. ${ }^{15}$ conducted the first study using bipolar radiofrequency for the treatment of chronic plantar fasciitis. In that limited number prospective study, the AOFAS score changed from a mean of 57.40 in the preoperative period to 88.50 in the postoperative period, with a minimum follow-up duration of 12 months without any notable postoperative complications.

The current study for radiofrequncy ablation of the plantar fascia is a little more conservative. Similar to the Weil et al. report, our patients were able to weight bear on the operated foot in a surgical boot immediately after the surgery. But our patients resumed normal shoes wear by 4 weeks after surgery as compared with 2 weeks in the Weil et al. ${ }^{15}$ series. A similar protocol has been reported by Sorenson et al. ${ }^{18}$

In comparison with historical controls, recovery time after open surgery of plantar fasciotomy has been documented to range from 4 to 8 months. ${ }^{16}$ As in many retrospective studies, we realize that our investigation was flawed by a number of methodological shortcomings that could threaten the validity of our conclusions. In particular, selection bias could have influenced our results, even though we described the outcomes in consecutive patients. Moreover, the treating surgeons determined the outcomes, although two outcome measures, namely the AOFAS score and VAS, with known subjective validity were used. ${ }^{19,20}$

\section{Conclusion}

We believe that percutaneous bipolar radiofrequency plantar micro-fasciotomy is an effective, minimally invasive, and safe surgical option for the treatment of recalcitrant plantar fasciitis (fasciosis) in adults of at least equal efficacy to the endoscopic procedure, which is also a highly successful procedure. It appears to be slightly safer than the endoscopic procedure due to fewer cases of nerve damage.

The limitations of this study include its retrospective nature as well as the non-randomized manner of treatment choice. The groups 
were thus slightly different demographically with the endoscopy group slightly but not significantly younger. Gender distribution was similar as well as the BMI but the micro-radiofrequency group were significantly more limited pre-operatively. At the latest follow-up results were similar in both groups. Due to these limitations the results should be validated in a double blind randomized study. It does seem to be possible to conclude that the micro-radiofrequency treatment is not inferior to the endoscopic release of the plantar fascia.

\section{Conflicts of interest}

None.

\section{Acknowledgements}

None.

\section{References}

1. Neufeld SK, Cerrato R. Plantar fasciitis: evaluation and treatment. J Am Acad Orthop Surg. 2008;16(6):338-346.

2. Irving DB, Cook JL, Young MA, et al. Impact of chronic plantar heel pain on health-related quality of life. J Am Podiatr Med Assoc. 2008;98:283-289.

3. Sobhani S, Dekker R, Postema K, et al. Epidemiology of ankle and foot overuse injuries in sports: A systematic review. Scand J Med Sci Sports. 2013;23:669-686.

4. Wearing SC, Hennig EM, Byrne NM, et al. Musculoskeletal disorders associated with obesity: a biomechanical perspective. Obes Rev. 2006;7(3):239-250.

5. Werner RA, Gell N, Hartigan A, et al. Risk factors for plantar fasciitis among assembly plant workers. $P M R$. 2010;2(2):110-116.

6. Fallat LM, Cox JT, Chahal R, et al. A retrospective comparison of percutaneous plantar fasciotomy and open plantar fasciotomy with heel spur resection. J Foot Ankle Surg. 2013;52(3):288-290.

7. Davies MS, Weiss GA, Saxby TS. Plantar fasciitis: how successful is surgical intervention? Foot Ankle Int. 1999;20(12):803-807.

8. Jerosch J, Schunck J, Liebsch D, et al. Indication, surgical technique and results of endoscopic fascial release in plantar fasciitis (E FRPF). Knee Surg Sports Traumatol Arthrosc. 2004;12(5):471-477.
9. Buchbinder R, Ptasznik R, Gordon J, et al. Ultrasound-guided extracorporeal shock wave therapy for plantar fasciitis: a randomized controlled trial JAMA. 2002;288(11):1364-1372.

10. Cavazos GJ, Khan KH, D'Antoni AV, et al. Cryosurgery for the treatment of heel pain. Foot Ankle Int. 2009;30(6):500-505.

11. Mahowald S, Legge BS, Grady JF. The correlation between plantar fascia thickness and symptoms of plantar fasciitis. J Am Podiatr Med Assoc. 2011;10(5):385-389.

12. Osborne HR, Breidahl WH, Allison GT. Critical differences in lateral $\mathrm{X}$-rays with and without a diagnosis of plantar fasciitis. J Sci Med Sport. 2006;9(3):231-237.

13. Ieong E, Afolayan J, Carne A, et al. Ultrasound scanning for recalcitrant plantar fasciopathy. Basis of a new classification. Skeletal Radiol. 2013;42(3):393-398.

14. Peterlein CD, Funk JF, Holscher A, et al. Is botulinum toxin A effective for the treatment of plantar fasciitis? Clin J Pain. 2012;28(6):527-533.

15. Weil L, Glover JP, Weil LS. A new minimally invasive technique for treating plantar fasciosis using bipolar radiofrequency: a prospective analysis. Foot Ankle Spec. 2008;1(1):13-18.

16. Kinley S, Frascone S, Calderone D, et al. Endoscopic plantar fasciotomy versus traditional heel spur surgery: a prospective study. J Foot Ankle Surg. 1993;32():595-603.

17. Brugh AM, Fallat LM, Savoy-Moore RT. Lateral column symptomatology following plantar fascial release: a prospective study. $J$ Foot Ankle Surg. 2002;41(6):365-371.

18. Sorensen MD, Hyer CF, Philbin TM. Percutaneous bipolar radiofrequency microdebridement for recalcitrant proximal plantar fasciosis. J Foot Ankle Surg. 2011;50(2):165-170.

19. Ibrahim T, Beiri A, Azzabi M, et al. Reliability and validity of the subjective component of the American Orthopaedic Foot and Ankle Society clinical rating scales. J Foot Ankle Surg. 2007;46(2):65-74.

20. SooHoo NF, Shuler M, Fleming LL. Evaluation of the validity of the AOFAS Clinical Rating Systems by correlation to the SF-36. Foot Ankle Int. 2003;24(1):50-55. 